\title{
Rehabilitation of severely resorbed maxilla with a hollow maxillary complete denture
}

\author{
A Bhandari ${ }^{1}$, SK Singh ${ }^{1}$, P Suwal ${ }^{1}$, PK Parajuli ${ }^{1}$, A Manandhar $^{2}$ \\ ${ }^{1}$ Department of Prosthodontics, ${ }^{2}$ Department of Conservative Dentistry \& Endodontics \\ College of Dental Surgery, BPKIHS, Nepal
}

\begin{abstract}
Rehabilitation of severely resorbed maxilla poses a clinical challenge for fabrication of a successful maxillary complete denture. The increased interridge distance often results in a heavy maxillary complete denture that further reduces the retention and stability of the denture. This case report describes the rehabilitation of an edentulous patient with resorbed maxillary and mandibular ridges and an increased interridge distance by hollow maxillary complete denture so as to reduce the weight of the denture which enhances the retention and stability of the denture.
\end{abstract}

Keywords: severely resorbed ridge, hollow maxillary complete denture, increased interridge distance.

\section{Introduction}

Residual ridge resorption is a complex biophysical process affected by various anatomic, prosthetic, functional and metabolic factors. ${ }^{1}$ Extreme resorption of the maxillary denture bearing area may lead to problems with prosthetic rehabilitation. These may be due to narrower, more constricted residual ridge as resorption progresses, decreased supporting tissues and a resultant large restorative space between the maxillary and mandibular residual ridge. Long lip length adds to this problem. This may result in a heavy maxillary denture that may further compound the poor denturebearing ability of the tissues and lead to decreased retention and resistance. ${ }^{2}$ Reducing the weight of a maxillary prosthesis, however, has been shown to be beneficial when constructing an obturator for the restoration of a large maxillofacial defect. ${ }^{3}$ The extensive volume of the denture base material in prostheses provided to patients with large maxillofacial defects or severe residual ridge resorption, reduction in prosthesis weight may be achieved by making the denture base hollow. Various weight reduction approaches have been achieved using a solid 3-dimensional spacer, including dental stone ${ }^{4}$ cellophane wrapped asbestos, ${ }^{5}$ silicone putty, ${ }^{2,6}$ or modelling clay ${ }^{7}$ during laboratory processing to exclude denture base material from the planned

Address for Correspondence

Dr. Arjun Bhandari

Junior Resident

Department of Prosthodontics, CODS, BPKIHS

Email:arjun906@hotmail.com hollow cavity of the prosthesis. This clinical report describes a technique for fabrication of a hollow maxillary complete denture in a patient with resorbed maxillary and mandibular ridges and increased interridge distance.

\section{Case report}

A 68 year old male patient reported to the Department of Prosthodontics, College of Dental Surgery, B. P. Koirala Institute of Health Sciences, Dharan, with the chief complaint of difficulty in chewing food and loose upper denture. History revealed that patient was edentulous for past 10 years. On examination, both maxillary and mandibular ridges were severely resorbed. His upper lip was long, the inter-ridge distance was more than normal. The previous denture of the patient was heavy and non retentive. Hence, it was decided to fabricate a new set of denture for the patient. The following treatment options for complete denture were available to the patient.

a. Implant supported complete denture.

b. Conventional Complete denture.

c. Hollow maxillary complete denture and conventional mandibular complete denture.

Pros and cons of all options were explained to the patient. He decided in favor of hollow maxillary complete denture and conventional mandibular complete denture as it was light in weight, inexpensive and non-surgical procedure. 


\section{Procedure for fabrication of hollow maxillary complete denture}

Maxillary and mandibular impressions were made followed by border molding with green stick compound (DPI Pinnacle tracing stick, Mumbai, India) and final impression with zinc oxide eugenol impression paste (DPI Impression paste, Mumbai, India).

The maxillomandibular relationship was recorded and transferred to the semi - adjustable articulator (Hanau Wide-Vue, USA) and the artificial teethAcry Pan, Ruthinium, India) were arranged in balanced occlusion.

Try in procedure was done.

\section{The special steps taken for the fabrication of hollow} maxillary complete denture were as follows:

Two split dental flasks with interchangeable counters were used for processing.

$\mathrm{V}$ shaped notches were made on the land area of the master cast at the anterior and the left and right posterior regions

Maxillary trial denture was flasked and dewaxed in the conventional manner using base 1 and counter 1 .

A shellac base plate Base plates, Dento Kem, India) was adapted over the denture base area of the master cast (base 1) and sealed with modelling wax (Modelling wax, Dento kem, India) covering the notches on the land area, then flasking was done by placing counter 2 over base 1. (Fig. 2)

A sheet of $2 \mathrm{~mm}$ modelling wax was adapted over the ridge lap surfaces of the denture teeth (counter 1) extending on the buccal and palatal surfaces at the level of the notches on the land area. The dough of dental plaster (Kaldent, Kalabhai, India) and pumicePumice powder, Samit,India), for easy removal after processing was placed in between the buccal and palatal wax sheet. The flasking was done by placing counter 1 over base 2. (Fig. 3)

Dewaxing and processing of 2 sets; base 1 , counter 2 and base 2 and counter 1 were done to obtain heat cure acrylic resin denture base (Trevalon, Dentsply, India) and acrylic teeth with heat cure resin on the buccal and palatal surfaces respectively. (Fig. 4)

Then the acrylic teeth with heat cure resin on the buccal and palatal surface was sealed with auto polymerising acrylic resin (RR, Dentsply, India) with the help of the notches on the land area to make a hollow denture.
The mandibular denture was processed with conventional manner.

Laboratory remounting was done to eliminate the processing errors.

The finishing and the polishing of the hollow maxillary and conventional mandibular complete denture were done.

The hollow cavity was verified by immersing the maxillary denture in water, no air bubbles were evident and denture floats in the water. (Fig 5)

The dentures were inserted in the patient's mouth and instructions were given.

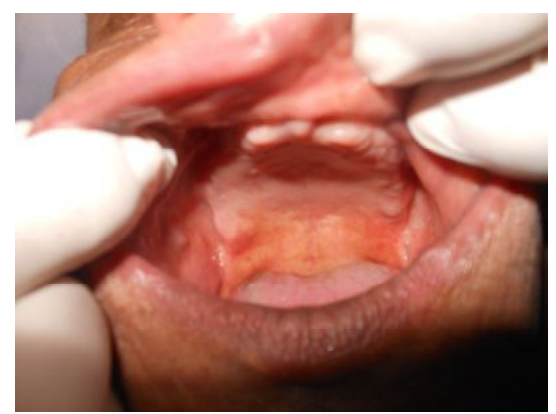

Figure 1: Intraoral view showing resorbed maxillary ridge.

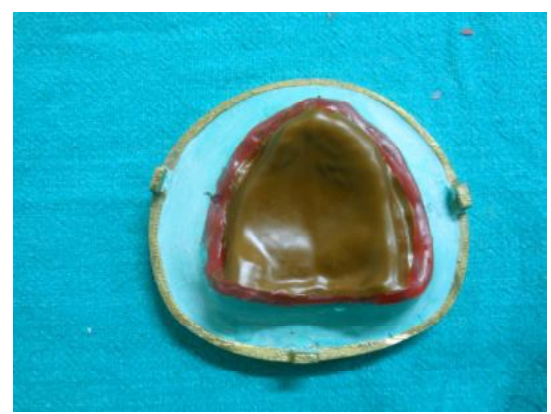

Figure 2: Adaptation of shellac base plate on the master cast on base 1 .

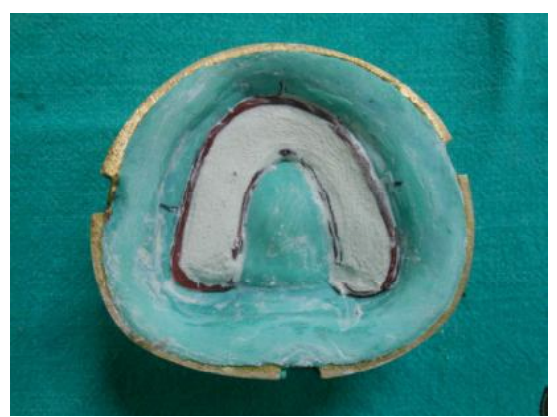

Figure 3: Dough of dental plaster and pumice on counter 1 . 


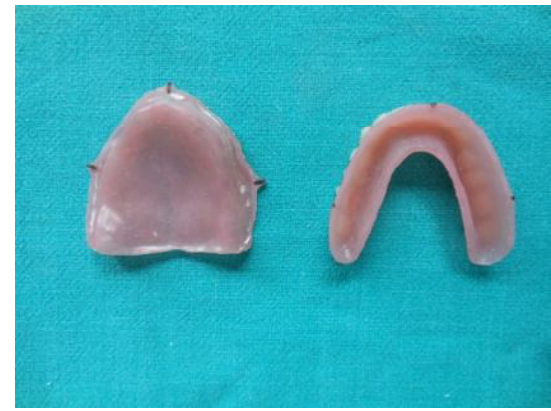

Figure 4: Two separate parts of maxillary denture after processing.

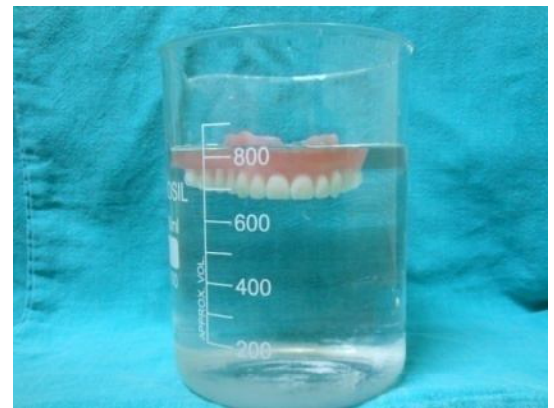

Figure 5: Light weight hollow denture.

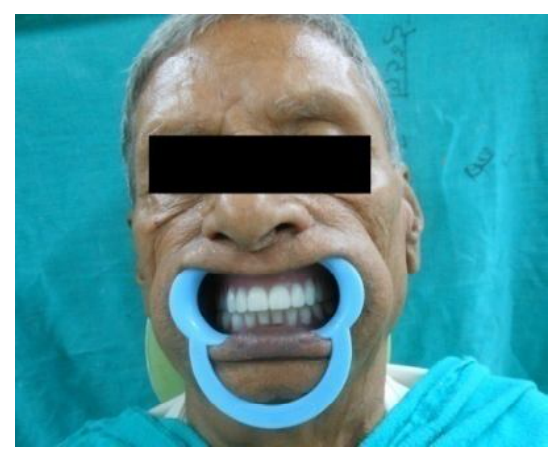

Figure 6: Patient after denture insertion.

\section{Discussion}

Rehabilitation of patient with severely resorbed ridges and long lip length is a challenge to the Prosthodontist. Even though, the choice for rehabilitation can be implant supported over denture, and ridge augmentation but many a times the patient who come with such a problem are geriatric patients with systemic illness, economic constrains, possess reluctance for a long duration treatment procedure and unwillingness for any kind of surgical procedure. Hence, the best way is to rehabilitate them with the conventional way. Apart, from modifying the impression technique to get maximum denture bearing area, modifying the type of denture may also be better accepted by the patient ${ }^{8}$. The advantages of hollow dentures are reduction in the excessive weight of the acrylic resin, resulting in the lighter prosthesis, decreases the load on the residual alveolar ridge making the patient comfortable. However, hollow denture is prone to fracture and requires more time to fabricate.

\section{Conclusion}

Hollow maxillary complete denture considerably reduces the weight of the prosthesis which in turn prevents transmission of detrimental forces which would otherwise be transmitted from a conventional heavy prosthesis to the underlying tissues. This ultimately results in increased retention and stability and up to some extent it is also possible to preserve the existing residual alveolar ridge.

\section{References}

1. Jahangiri L, Devlin H, Ting K, et al: Current perspectives in residual ridge remodeling and its clinical implications: a review. The Journal of Prosthetic Dentistry 1998;80:224-237.

2. O'Sullivan M, Hansen N, Cronin RJ, Canga DR: The hollow maxillary complete denture: A modified technique. The Journal of Prosthetic Dentistry, 2004; 91(6):591-594.

3. McAndrew MS, Rothenberger S, Minsley GE: An innovative investment method for the fabrication of a closed hollow obturator prosthesis The Journal of Prosthetic Dentistry. 1998;80:129-132.

4. Fattore LD, Fine L, Edmonds DC. The hollow denture: an alternative treatment for atrophic maxillae. The Journal of Prosthetic Dentistry. 1988;59:514-516.

5. Worley JL, Kniejski ME: A method for controlling the thickness of hollow obturator prostheses. The Journal of Prosthetic Dentistry. 1983;50:227-229.

6. Gardner LK, Parr GR, Rahn AO: Simplified technique for the fabrication of a hollow obturator prosthesis using vinyl polysiloxane. The Journal of Prosthetic Dentistry. 1991;66:60-62.

7. DaBreo EL.Alight-cured interim obturator prosthesis. Aclinical report. The Journal of Prosthetic Dentistry. 1990;63:371-373.

8. Kalavathy N, Shetty MM, Premnath, Pawashe K, Patel RKV: Hollow mandibular complete denture A case report. SRM University Journal of Dental Sciences, 2010;1(3): 243-246. 